\title{
La educación artística en la Escuela de Bellas Artes de Barcelona durante el siglo XIX. El caso de la escultura
}

\author{
The Artistic Education at the Barcelona School of Fine Arts \\ during the $19^{\text {th }}$ century. The case of Sculpture
}

\author{
Cristina Rodríguez-SAMANiEgo \\ Universidad de Barcelona. \\ cristinarodriguez@ub.edu
}

Recibido: 11 de noviembre de 2012

Aprobado: 13 de diciembre de 2012

\begin{abstract}
Resumen
El presente artículo propone una visión integradora de la evolución del aprendizaje de la escultura en la Escuela de Bellas Artes de Barcelona durante el siglo XIX, cuando se moderniza la docencia y se sientan las bases para la enseñanza artística contemporánea. Para ello, se analiza la estructura curricular de la asignatura, los materiales docentes empleados en ésta, la faceta pedagógica y planteamientos estéticos de sus profesores, y se profundiza en cómo el debate tradición - modernidad impregna los discursos estéticos producidos en el marco de la Academia de Bellas Artes de la ciudad y se transmite a la práctica de la disciplina. Este artículo, elaborado a partir de documentación primaria y fruto de un proyecto de investigación financiado, presenta información inédita sobre el aprendizaje oficial de la escultura en la época tratada. Los datos aportados permiten establecer una comparativa con el sistema actual y plantear una evolución de la enseñanza de la escultura en nuestro país.
\end{abstract}

Palabras Clave: escultura, siglo XIX, Escuela de Bellas Artes, pedagogía del arte, clasicismo.

Rodríguez-Samaniego, C. (2013): La educación artística en la Escuela de Bellas Artes de Barcelona durante el siglo XIX. El caso de la escultura. Arte, Individuo y Sociedad, 25(3) 494-507

\begin{abstract}
Information processing and memory have an important role in stimulating, encoding and recall of advertising stimuli. Through an experimental study the present article seeks to identify the impact on short-term memory of visual rhetorical figures, comparing real advertisements and works of artist Magritte intervened and presented as branded advertisements. The hypothesis states that rhetorical figures present in advertising designs should have a greater recall effects than artistic works. The results confirm the thesis, showing that the functional use of rhetorics as planned advertising elements achieve higher a recall rate compared to its use in Magritte's art.
\end{abstract}

Key Words: sculpture, $19^{\text {th }}$ century, School of Fine Arts, Art pedagogy, classicism.

Rodríguez-Samaniego, C. (2013): The Artistic Education at the Barcelona School of Fine Arts during the $19^{\text {th }}$ century. The case of Sculpture. Arte, Individuo y Sociedad, 25(3) 494-507

Sumario: 1. Introducción, 2. Escultura: materiales didácticos y estructura docente, 3. Profesorado: obra, docencia y planteamiento estético, 4 . Discursos: debate en torno al clásico y a la copia, 5 . A modo de conclusión . Referencias. 
Este artículo recoge resultados de la investigación "Escultores de la Academia de Bellas Artes de Barcelona (1849-1900): dinámicas, docencia e internacionalización = Sculptors from the Barcelona Academy of Fine Arts (1849-1900): dynamics, teaching and internationality" financiada por el subprograma Juan de la Cierva (MICINN. Ref: JCI-2010-06550) y ha recibido el apoyo del grupo de investigación GRACMON (Universidad de Barcelona), inscribiéndose en su línea de investigación financiada "El otro siglo XIX" (MICINN. Plan Nacional de I+D+I. Ref: HAR2010-16328/ HIST-ARTE).

\section{Introducción}

La segunda mitad del siglo XIX es uno de los momentos más interesantes de la historia de la educación artística en España, un periodo rico y paradójico, en el que se incrementa considerablemente el número de Academias de Bellas Artes y de Escuelas dependientes de éstas, modernizándose su funcionamiento, estructura y tipo de docencia impartida, pero en el que, a la vez, se sientan las bases para su decadencia posterior, entrado ya el siglo XX. Se trata, pues, de una época de esplendor y desgaste, de luces y sombras, imprescindible cara a la comprensión del desarrollo de las artes en nuestro país. En el caso de la Academia de Barcelona, ésta es una etapa en la que sus relaciones con la Escuela de Bellas Artes se hacen más cercanas y complejas y en la que ambas instituciones sufren modificaciones sustanciales, que derivarán en una separación definitiva de las dos entidades en 1901, separación de la que surgen las actuales Escuela Superior de Diseño y Arte (Escola Llotja), y la Facultad de Bellas Artes de la Universidad de Barcelona. En la segunda mitad del siglo XIX, el aprendizaje de las artes y, entre ellas, de la escultura, se ve sometida a notables cambios sobre todo en cuanto a la formación teórica y a la estructura curricular, cambios que sentarán las bases para la enseñanza de la disciplina en la actualidad.

A finales de 1849, la Escuela de Bellas Artes, que ya existía, pasó de depender de la Real Junta de Comercio de Barcelona a estar bajo el control de la recién creada Academia Provincial de Bellas Artes de la ciudad (Por Real Orden de 31 de octubre de 1849). La historia de la Junta y la de la Escuela gratuita de Diseño que ésta había puesto en funcionamiento en 1775, han sido ya tratadas por diversas fuentes (Martinell, 1951; Ruiz Ortega, 1999; Subriachs, 1994). Todas ellas coinciden en subrayar el carácter eminentemente industrial que tuvieron las enseñanzas de la escuela en sus inicios y en cómo, poco a poco, el centro fue dejando de lado su carácter primigenio de formador exclusivo de trabajadores para empresas. De esta manera, daba cabida a la vez a personas que ansiaban ganarse la vida como artistas, en la acepción actual del término. El último tercio del siglo XVIII se habían reestructurado y ampliado las enseñanzas dependientes de la Junta, y se añadieron a las de Dibujo las de Nobles Artes, a fin de encauzar correctamente las vocaciones "artesanas" y "artísticas" (ó "de aplicación" " “superiores", empleando la terminología de la época, que mantendremos a partir de ahora) de los alumnos que a ellas concurrían.

Esta división tan significativa de las enseñanzas de la escuela tuvo continuidad a pesar de la reforma que siguió a la creación de la Academia provincial. De hecho, llega hasta nuestros días, teniendo las enseñanzas de Aplicación su paralelo en la 
Escuela de Diseño y Arte, y las Superiores el suyo en la Facultad de Bellas Artes de Barcelona. Los dos caminos formativos incorporaban asignaturas vinculadas a la escultura, aunque en el caso de los de Aplicación éstas incluían Vaciado, Talla y Nociones de metalistería, mientras que en los Superiores se impartía la escultura de tipo más creativo, en el sentido convencional del término. En general, los escultores profesionales formados en aquella época cursaban únicamente los estudios Superiores, por lo que, en este artículo, nos centraremos en la enseñanza de la escultura en los Superiores, aunque también se hará mención de algún aspecto relativo a los de Aplicación.

\section{Escultura: materiales didácticos y estructura docente}

La Escultura era una asignatura considerada de orden práctico en la Escuela de Bellas Artes durante el período que nos ocupa. Esto implicaba que el alumno que la cursaba debía apoyar los conocimientos adquiridos en la clase con otras materias de carácter teórico, como Anatomía, Teoría e historia de las Bellas Artes, y Perspectiva, y además compaginarlo con el aprendizaje del dibujo (del antiguo y del natural). En la formación de un buen escultor, el dibujo era una base imprescindible, la capacidad de relacionar la obra con la tradición artística resultaba esencial, y se entendía que el conocimiento de las formas y la estructura y funcionamiento internos del cuerpo humano se hacían imprescindibles. Un examen de ingreso servía para valorar las capacidades iniciales del alumno en este sentido. Por otra parte, casi todas las asignaturas se encontraban divididas en secciones diferentes; a medida que el estudiante aprendía y mejoraba, pasaba de la más elemental a la más exigente y compleja. Aunque no había un régimen de permanencia como el que existe en la actualidad, se consideraba que si no se superaban las secciones elementales en cinco años, el alumno no podía volverse a matricular (Mns. 296.3.24, RACBASJ).

Durante el curso, los estudiantes debían presentarse a pruebas periódicas de las asignaturas prácticas, que se convocaban cada quince días o cada mes, con el objetivo de obtener los llamados "pases de clase" y poder avanzar a un nivel superior de la materia. En dichas pruebas, se analizaban los méritos de los trabajos presentados y, si eran satisfactorios, se otorgaban menciones honoríficas. Eran necesarias cuatro menciones como mínimo para poder subir de nivel en la clase de Escultura y, a menudo, se requerían cinco, aunque la normativa de 1851 exigiese sólo tres (Mns. 296.3(24), RACBASJ ). A finales de curso, se ponderaba la evolución del alumno y se valoraba la posibilidad de que avanzara si no lo había hecho ya antes. Una vez aprobados todos los niveles, el estudiante había superado la asignatura y obtenía el correspondiente diploma. En este sentido, el desarrollo curricular de Escultura era distinto del de las clases teóricas, en las que había censuras trimestrales, exámenes parciales y ordinarios finales, además de los extraordinarios. La tiología de los exámenes de las asignaturas teóricas se consolidó en la Escuela a partir del curso 1860-61 (Mns. 251.1.6, RACBASJ).

Resulta interesante constatar que, en el caso de la Escultura, el primer nivel - la primera clase -, era la de Escultura del antiguo, es decir, copia de piezas maestras de la historia del arte. Éstas eran sobre todo clásicas, tanto en el estilo y como en la procedencia. El hecho de que Antiguo fuera la primera unidad en la que se dividía 
la materia, nos permite enfatizar el peso que la tradición y el clásico tenían en esta enseñanza, un peso que se mantuvo, aunque cuestionado, hasta finales de siglo. A continuación, superado el Antiguo, se pasaba a Escultura del natural, donde se copiaba ante modelo vivo. La escuela disponía de varios hombres que ejercían de modelo y que posaban para las clases, no sólo en la de Escultura. El último nivel era el de Composición, donde se trataba de trabajar la creatividad personal en función de los conocimientos adquiridos a través de la copia. En este punto, también se hacía hincapié en elementos y recursos formales concretos, como los pliegues de vestimenta. Vale la pena apuntar que esta subdivisión de la asignatura en tres secciones existía ya a principios de siglo (Reglamento de la Escuela Gratuita..., 1839).

Durante la segunda mitad del XIX, Escultura se impartió siempre en el segundo piso del edificio de Lonja, aunque el aula en la que se enseñaba cambió de emplazamiento varias veces. Justo después de la reestructuración de 1849 , no existía un espacio reservado únicamente a la enseñanza de la disciplina, sino que compartía aula con Dibujo y, probablemente, también con Anatomía. Esta era una organización lógica en ese momento, sobre todo si tenemos presente la importancia que tenía el dibujo y el conocimiento de las formas del cuerpo humano en el aprendizaje artístico en la Escuela. En 1875, Escultura y Dibujo seguían impartiéndose juntas (aunque Escultura disponía también de un espacio en el Museo del centro), y Anatomía se daba en la tercera planta del edificio (plano 3250D, RACBASJ) (Fig.1). A finales de siglo, esta situación había ya cambiado y Escultura ocupaba dos espacios independientes contiguos, en el ángulo entre los actuales Paseo de Isabel II y Pla de Palau, donde antiguamente estaba la sala de profesores. A partir de entonces, la clase se fue ampliando, pero se mantuvo en el mismo lugar, separada de otras asignaturas (Escuela Superior de Artes é Industrias..., 1903) (Fig.2).

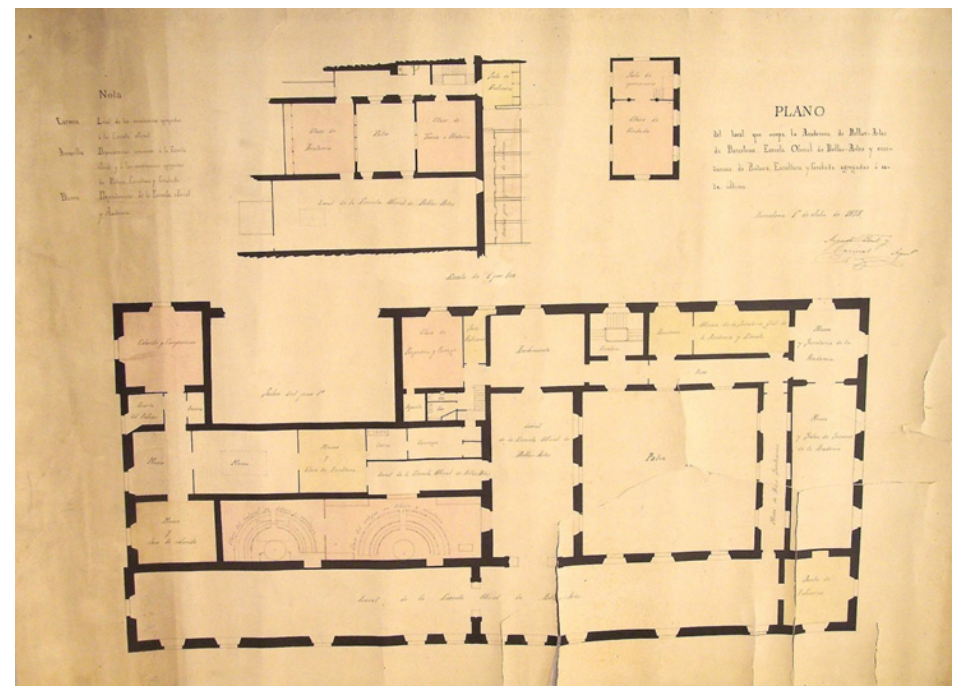

Figura 1. Plano de la Escuela y Academia de Bellas Artes de Barcelona, 1875. ( $\mathrm{n}^{\circ}$ inventario 3250D, RACBASJ). Pueden apreciarse los dos espacios contiguos con gradería, reservados al Natural y al Antiguo en Dibujo y Escultura. Adyacente a éstos, el Museo y aula de Escultura. 


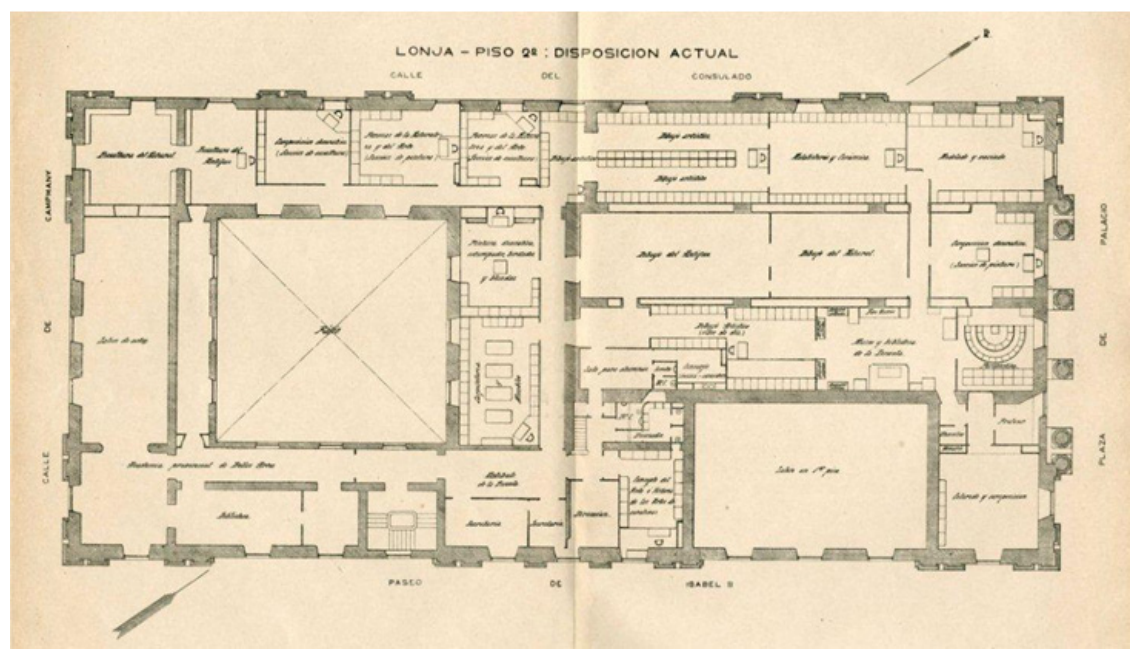

Figura 2. Plano de la Escuela y Academia de Bellas Artes de Barcelona, c.1903.

(Escuela Superior de Artes é Industrias..., 1903). Orientación del plano invertida respecto a la ilustración anterior. Los cambios en la distribución del espacio de la Escuela son notables y dan cuenta de la voluntad de modernización de la docencia. En el extremo superior izquierdo, en paralelo a la Calle del Consulado, se hallan las aulas del Natural, Antiguo, y las de las nuevas asignaturas Composición decorativa, Formas de la naturaleza y del arte, etc.

Los inventarios de la institución nos permiten comprobar cómo, en esta época, en el aula del Antiguo había gran cantidad de estatuas antiguas en yeso, fragmentos anatómicos también en este material, dibujos y estatuas copia del antiguo de alumnos premiados, todo alrededor de un anfiteatro de madera frente a una tarima redonda y giratoria donde se colocaba la obra a copiar. En unos armarios, se conservaba la ropa de maniquí del Antiguo: cascos y corazas de latón, escudos, sables, calzado, pelucas, etc. Los alumnos de escultura disponían, además, de atriles y trípodes giratorios en los que situar sus ejercicios y farolillos de gas que se podían desplazar por la clase (Mns. 295.2.1.19, RACBASJ ). De hecho, la asignatura de Escultura - Natural y Antiguo-se hacía por la tarde, en la época de Damià Campeny i Estrany (1771-1855) de las 16 a las $18 \mathrm{~h}$ y, después, en las épocas de Andreu Aleu i Teixidó (1832 -1901), Joan Roig i Solé (1835-18) y Agapit Vallmitjana i Barbany (c.1832 -1905), de las 19 a las $21 \mathrm{~h}$. Entendemos, por tanto, que durante muchos meses al año se trabajaba con luz artificial.

Por otro lado, la clase del Natural, en dibujo y escultura, era similar a la del Antiguo, con una grada de las mismas dimensiones y capacidad, paneles donde se colgaban obras de alumnos premiados, y yesos didácticos. También había un buen número de bajorrelieves de composición. En cuanto a la ropa de maniquí, en el aula del Natural había principalmente tejidos, para la práctica del pliegue. Finalmente, 
el espacio disponía de todo lo que el modelo que posaba pudiera necesitar: desde un espacio donde cambiarse hasta un pedestal cómodo donde situarse. A partir de la década de 1870, con la separación de las asignaturas en aulas diferentes, Escultura se enseña en un espacio sin gradería, pero ve aumentada la colección de yesos didácticos a disposición de los alumnos (Academia Provincial de Bellas Artes de Barcelona, ${ }^{1897)}$.

Como materiales didácticos para la primera sección de la asignatura de Escultura, en la Escuela se empleaban principalmente dibujos, grabados y vaciados en yeso de obras antiguas, muchos de los cuales se encontraban, como acabamos de ver, en las aulas. El antiguo era entendido fundamentalmente como sinónimo de clásico; la documentación que se conserva en el archivo de la Academia hace patente que la colección de piezas usadas para las clases y las del fondo del Museo eran, principalmente, reproducciones de esculturas o de motivos escultóricos griegos y latinos. Los yesos se encargaban a menudo al profesor de Vaciado y los grabados a los profesores de Dibujo o de Grabado, pero a veces también se adquirían en el extranjero. Las copias de obras renacentistas, de espíritu clásico, ocupaban un segundo lugar, seguidas de las de otras procedencias, siempre antiguas y a menudo compradas en Inglaterra. Dada la intensidad del uso que se daba a estos materiales, los encargos y adquisiciones eran constantes (Fig.3). Mucho más económica y versátil que el grabado, la fotografía se fue imponiendo paulatinamente como herramienta de aprendizaje de la Escultura, sobre todo en el último tercio de siglo XIX. En aquella época, la biblioteca de la Escuela compró fotografías sueltas y álbumes de fotografías que dinamizaron las enseñanzas de la asignatura.

Asimismo, la biblioteca contaba también con libros ilustrados. Dentro de esta línea, podemos destacar las publicaciones de arquitectura, de historia y teoría del arte, de anatomía, de elementos decorativos y de dibujo de figura, que permitían completar la formación de los futuros escultores y que servían al mismo tiempo para aprender el dibujo y para las asignaturas teóricas (Fig.4). Muchos de ellos se compraban en el extranjero, o bien se encargaban a libreros de Barcelona que aceptaban pedidos internacionales.

Finalmente, mencionaremos que, tanto cara al aprendizaje de la escultura como para ilustrar al resto de los estudiantes, también se empleaba esculturas creadas por los profesores de la casa. Los alumnos las podían observar en sus emplazamientos del Museo de la Academia (donde se podía ir a dibujar, previa cita) y en los espacios comunes de la escuela. La Escuela poseía, durante el siglo XIX, una colección muy abundante de yesos, que reponía y renovaba con frecuencia. A los yesos expuestos, deben sumarse los que se hallaban en los almacenes del piso superior, a la espera de ser usados o reconvertidos. De toda esta colección, en la actualidad se conserva muy poco, el Museo de la Reial Acadèmia Catalana de Belles Arts de Sant Jordi cuenta con algunas piezas, y el Museo Frederic Marès de Barcelona alberga en su fondo un conjunto de fragmentos anatómicos procedentes de la Escuela. 


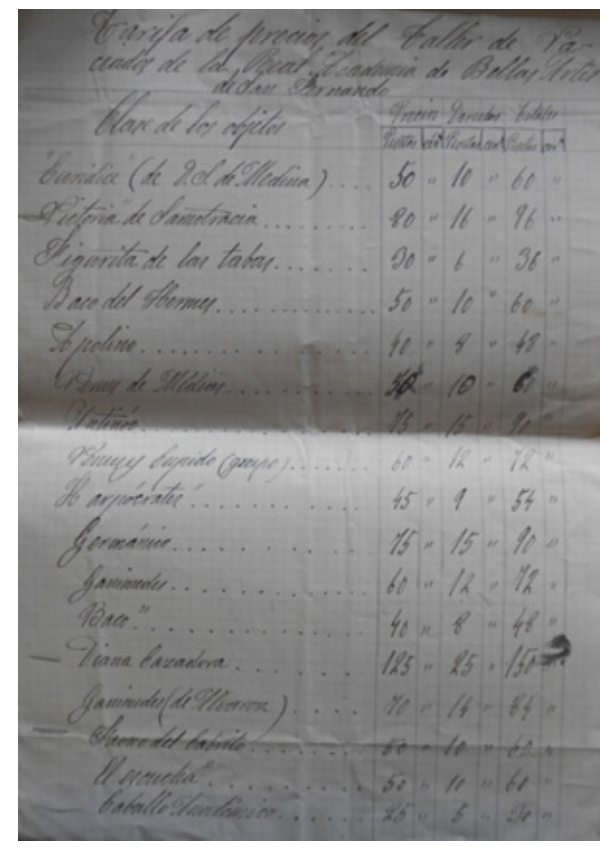

Figura 3. Lista de precios del taller de Vaciado de la Real Academia de Bellas Artes de San Fernando, c. 1890.(RACBASJ). Presupuesto propuesto a la Academia de Bellas Artes de Barcelona.

El estilo de estas obras correspondía, evidentemente, a los gustos estéticos imperantes entonces. En ellas, se hace patente la primacía de lo clásico, sobre todo en las creadas durante la primera mitad del XIX, cuando desde la Academia se fomentaba y protegía el ideal neoclásico. El hecho de que muchas de éstas hubieran sido creadas en el marco del Premio de Roma (algunas habían hecho ganar el concurso a sus autores, otras habían sido enviadas a la Academia desde la capital italiana en el transcurso de la estancia fruto de la beca), da razón, en parte, del estilo de la colección. Sin embargo, a lo largo de la segunda mitad del mil ochocientos, penetraron en Cataluña movimientos europeos, principalmente el romanticismo idealista, nazareno; tendencias que terminaron manifestándose en las obras de los profesores de la Escuela y que, en consecuencia, fueron transmitidas también a sus alumnos. Por su parte, el simbolismo y el modernismo finiseculares tuvieron una aplicación desigual y tardía a la escultura, y no disfrutaron de cierta presencia en la Escuela hasta entrado el siglo XX (Subirachs, 1994, pp.27-52).

$\mathrm{Al}$ ser la Escultura una asignatura de carácter práctico, no se hacía necesaria la existencia de un programa concreto de la materia, a diferencia de lo que sucedía con las teóricas. Sin embargo, la orientación pedagógica de las clases puede inferirse a través tanto de los discursos de los profesores y académicos -un tema que se tratará a continuación-, como del material didáctico que acabamos de analizar, elementos de los que se desprende una clara primacía del clásico, de la copia de modelo antiguo. Sin embargo, a medida que avanzó el siglo, el peso del Natural 
y de la Composición, per se, fueron cogiendo protagonismo. Este hecho se hizo particularmente patente con motivo de la reforma de 1892, cuando se replanteó el enfoque de las enseñanzas de la Escuela para fomentar el trabajo sobre el Natural. En lo tocante a Escultura, aparecieron las clases de Composición decorativa y la de Formas de la naturaleza y del arte (Fig.2), las cuales, junto con las de Antiguo y Natural se convirtieron en talleres donde los alumnos podían trabajar cuando lo consideraran necesario (Escuela Superior de Artes é Industrias y Bellas Artes de Barcelona, 1903: 13, 16-17), un planteamiento parecido al que rige hoy en la Facultad de Bellas Artes.

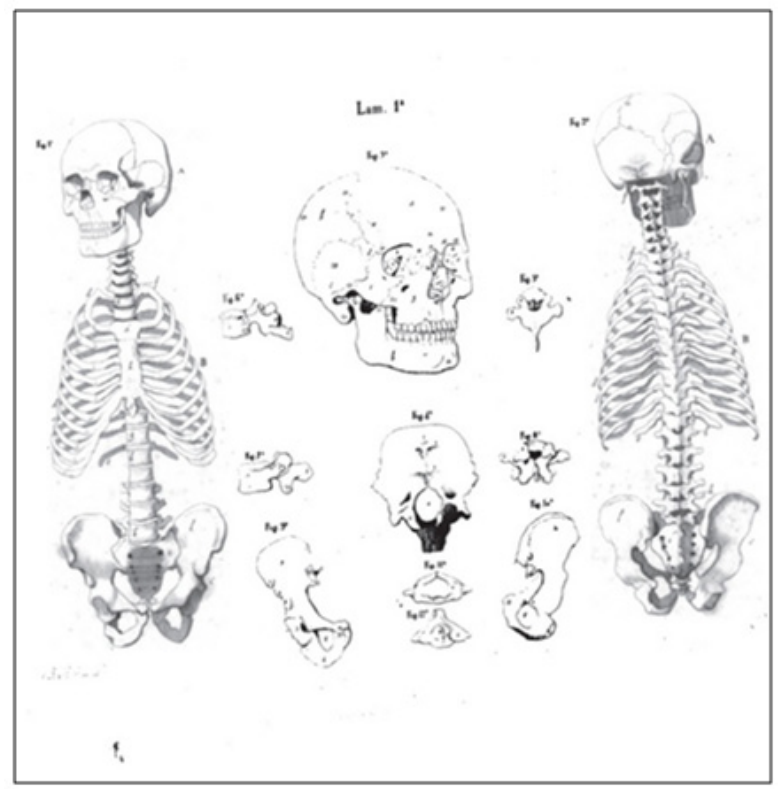

Figura 4. Ilustración de la obra de Gerónimo Faraudo Estudios de la Historia natural del hombre aplicados á la pintura y escultura ó sea Lecciones de Anatomá y fisiología artísticas dadas en la Academia de Bellas Artes de Barcelona, 1852 (RACBASJ).

\section{Profesorado: obra, docencia y planteamiento estético}

Entre las obras que se han conservado en el Museo de la Academia, descubrimos los nombres de escultores importantes en la España del siglo XIX, la mayor parte de los cuales fueron también docentes de la Escuela durante el período que nos ocupa. El ya mencionado Damià Campeny, fue quien más contribuyó a la colección, sobre todo con obras realizadas en Roma durante el primer tercio del XIX. Campeny fue uno de los maestros más longevos de la institución, dado que impartió clases unos treinta años, hasta su defunción en julio de 1855. A diferencia del resto de escultores profesores de su época, la figura y la producción de Campeny han sido bien estudiadas y son bastante conocidas hoy en día (Cid, 1998). Su filiación con la estética neoclásica resulta evidente, pues contribuyó a su importación desde Italia y la promocionó tanto 
desde su faceta de creador como la de maestro. Sin embargo, el neoclásico no es el único material que exploró Campeny. En nuestra opinión, a Campeny le debemos, además, un paso adelante hacia la consecución del denominado "estilo académico" o "academicismo" en la escultura catalana del XIX. Precisamente porque esta terminología ha sido a menudo utilizada de forma vaga y poco concisa en relación a la escultura catalana y española, resulta imprescindible acotarla. La académica es una propuesta formal y estética vinculada al mundo de las Academias de Bellas Artes, que preconiza una aproximación objetiva, regular, sobria, no naturalista y de aspiración conservadora a la creación plástica, con una selección temática más bien reducida, en la que, en términos generales, la anécdota, la expresión sentimental y la narrativa tienen poca cabida (Fig.5). En la concreción del estilo académico, los postulados estéticos de las Academias de París y Roma ejercieron una influencia esencial. La relación del bagaje clásico - griego y latín-con el academicismo catalán del XIX debe tenerse en cuenta, principalmente en términos iconográficos, aunque, por supuesto, las obras académicas de la época que nos ocupa no representan únicamente sujetos vinculados a la iconografía clásica, sino que abarcan un abanico mucho más amplio de motivos e imágenes. El academicismo podría ser entendido, también, como una forma de perpetuar el clásico a nivel formal, y no tanto a nivel estético. Desgastada ya la advocación del clásico típica del neoclasicismo, el academicismo recupera y reelabora su planteamiento plástico, convirtiéndose en un lenguaje práctico, digerido, universal, lejos ya de los grandes debates filosóficos y artísticos sobre el tema aparecidos en la segunda mitad del siglo XVIII y principios del XIX.

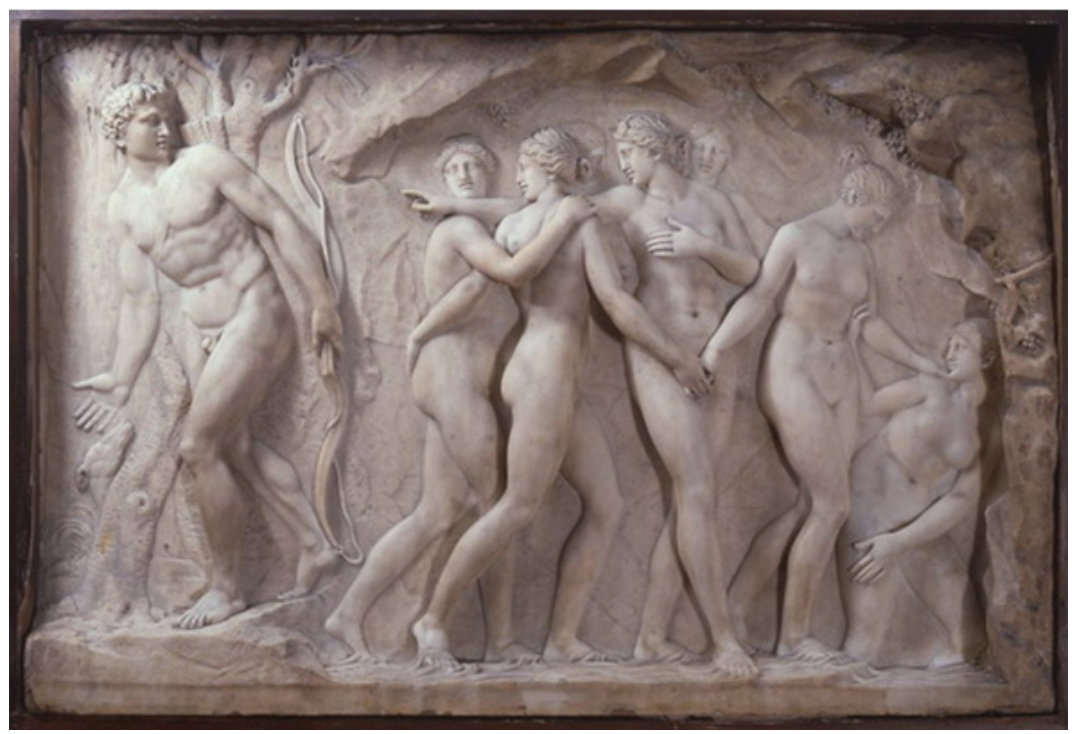

Figura 5. Damià Campeny, Diana y sus ninfas bañándose, sorprendidas por Acteón, c.1798-1799, mármol (RACBASJ no inventario 302E). 
Por otra parte, y aunque pueda parecer paradójico, Damià Campeny también es uno de los responsables, en última instancia, de la irrupción de las nuevas corrientes estéticas en la escultura del XIX en nuestro país, especialmente en Cataluña. Obras como el Galo Herido o Degollación de San Juan Bautista nos permiten ya hablar de un Romanticismo incipiente, patente en la gestualidad, el dinamismo y la expresividad de los personajes que las componen. El ejemplo de Campeny fue seguido por las dos generaciones de escultores que formó en la Escuela y en su taller, sentando así las bases para la evolución de la disciplina y de su enseñanza en la segunda mitad del XIX. Los profesores que lo siguieron en la docencia de la escultura perpetuaron la primacía del estilo académico hasta el cambio de siglo y, al mismo tiempo, estuvieron abiertos a la llegada de los estilos nuevos que venían de Europa, adaptándolos a la naturaleza y las particularidades de la práctica artística local. Durante los últimos años de Campeny, cuando sus dificultades motoras se agravaron, el rol de sus ayudantes en la clase de Escultura se hizo más importante. Dos de los asistentes del maestro continuaron con la difusión de las corrientes románticas y de la estética académica en la Escuela durante las décadas centrales del XIX. Josep Bover i Mas (1802 -1866) cumplía con las labores de asistente de Campeny ya desde 1833, cuando el mataronés era todavía docente de Dibujo. Bover, en la cantera de la Escuela hasta 1853, nunca impartió como titular la asignatura de Escultura, pero se dedicó a esta disciplina de forma profesional, con una carrera que le llevó por toda España. Bover continuó con la línea académica propia de Campeny, maduró y consolidó este estilo y lo trasladó a la obra pública, principalmente la estatuaria. Por otra parte, de este autor son también piezas de carácter plenamente romántico, como el Gladiador herido, una de las más importantes del fondo de escultura que los alumnos de la Escuela de Bellas Artes tenían a su disposición y que se empleaba continuamente para las clases, de Escultura, Dibujo y Vaciado (Mns. 295.2.2.2, 295.2.1.19; 75.10.5, RACBASJ).

También ayudante de Campeny fue Andrés Aleu, quien ocupó la dirección de Escultura a la muerte del maestro. Aleu, además, entró a formar parte de la sección de esta disciplina en la Junta de la Escuela, lo que le permitió ejercer su voto en los dictámenes de la Academia y expandir así el gusto y preferencias estéticas de la institución en el campo de la escultura en espacios públicos laicos, religiosos e, incluso, privados. Recordemos que los profesores de la Escuela, además de sus tareas profesionales y docentes, formaban parte de comisiones dedicadas a juzgar la idoneidad de las obras artísticas de carácter público que se debían llevar a cabo en la ciudad y zona de influencia de la Academia. Los proyectos de obras debían ser sometidos a la aprobación de la Academia y, si procedía, ésta dictaminaba el aprobado o bien requería que se hicieran modificaciones. Aunque no se conserve ninguna obra suya actualmente en la Academia, Aleu fue un escultor prolífico, recordado hoy principalmente gracias a su Sant Jordi, de la fachada del Palacio de la Generalidad en Barcelona. El romanticismo de Aleu y de Bover se caracteriza por la representación de personajes vinculados a la historia y el imaginario nacional catalán, una línea de trabajo que se consolidó en la década de 1860 y que fue muy popular entre los escultores de la época que nos interesa. También destacó por la realización de esculturas de corte más naturalista, particularmente retratos de personajes de la historia más reciente, como el Monumento al Marqués del Duero de Madrid. La 
historiografía del arte de nuestro país ha situado este tipo de piezas dentro de la corriente del realismo internacional, aunque hay que tener presente que en la escultura catalana del XIX no se puede hablar de un realismo con la carga crítica y de denuncia social que este movimiento tuvo en otros países europeos, como es el caso de Francia.

A partir de la década de 1870, con los profesores Joan Roig o Agapit Vallmitjana i Barbany, se abandona la estética romántica en la práctica de la escultura, en favor de apuestas más naturalistas, que triunfaron en la Escuela y la Academia durante el último tercio de siglo. El rol de la saga de los Vallmitjana en este proceso ha sido ya estudiado (Rodríguez Codolà, 1946), aunque probablemente merezca ser revisado. El clásico perdió empuje, aunque continuó presente en la escultura academicista y, en especial, en la monumental y conmemorativa.

\section{Discursos: debate estético en torno al clásico y a la copia}

A raíz de sus nombramientos, los nuevos académicos, profesores o no, solían hacer un discurso que se publicaba y distribuía. También se hacían con motivo de la inauguración del curso escolar. Aunque no se han conservado discursos leídos por académicos escultores del siglo XIX, el análisis de las disertaciones de profesores coetáneos de otras disciplinas nos permite entender cuáles eran los parámetros estéticos que regían la docencia entonces, y discernir qué peso tenía el clásico, el idealismo, y qué espacio se dejaba al natural y a la creatividad individual (Freixa i Serra, 2008). En este momento, previo al surgimiento de las vanguardias históricas, la figuración era un requisito que se daba por descontado en cualquiera de las materias impartidas en la Escuela, incluida la Escultura, un presupuesto superado hoy en día. El imperativo que los alumnos contaran con una sólida base clásica, tanto a nivel de teoría y estética, como en el aprendizaje práctico, se mantuvo hasta 1900, pero la filiación formal con el clásico, defendida hasta mediados de siglo, fue paulatinamente puesta en cuestión a medida que se acercaba el final del siglo.

Durante las últimas décadas del XIX, el ejemplo de la escultura clásica grecolatina siguió siendo esencial, preponderante, si bien, entre los profesores había cierta coincidencia en prevenir que el estudio del movimiento, de la anatomía y de los volúmenes de los cuerpos también eran factores imprescindibles cara a la construcción de una buena escultura. Por supuesto que cada maestro enfatizaba la trascendencia de aspectos vinculados a su asignatura, pero, aún así, defendían la necesidad de matizar la primacía del clásico a través del estudio del natural. Gerónimo Faraudo i Condeminas (1823-1886), profesor de Anatomía, por ejemplo, se mostraba convencido en su discurso de 1863 que los escultores jóvenes debían fijarse en la obra de Fidias, Policleto, Praxíteles, Eufránor y Lisipo, porque éstos representaban la justeza del ideal y la plenitud del objeto, pero también de la naturaleza; subrayando la importancia de la adecuación del espíritu a la forma y el cuidado en la caracterización (D-1863. Discursos (per anys) 1859-1915, RACBASJ). Por su parte, Tiberio Ávila, quien subsitiyó a Faraudo en la cátedra de Anatomía, dirigió un discurso breve a los alumnos premiados con pensiones en 1896, en el que hizo un análisis de la situación artística de la época, aprovechando para orientar a los alumnos sobre cómo enfrentarse a ésta. Ávila preconizaba el esfuerzo y la perseverancia, el equilibrio entre lo real y lo ideal, entre la imaginación y la razón (D-1896. Discursos (per anys) 1859-1915, 
RACBASJ), dejando la puerta abierta a planteamientos creativos más personales y alejados de la omnipresencia de la copia.

El caso de Josep de Manjarrés i Bofarull (1816-1880), profesor de Teoría e historia de las Bellas Artes y director de la institución durante un tiempo, es aún más revelador. En 1859, en sesión pública, Manjarrés apuntaba a la importancia de la experiencia y del razonamiento por encima del principio de autoridad y el dogmatismo en la Escuela, y defendía la importancia del talento y del genio. Se mostraba completamente en contra del principio de imitación en el aprendizaje. A su juicio, de los maestros se podía aprender, pero nunca copiar los recursos. Pese a lo contemporáneas que sus opiniones puedan parecer, debemos tener en cuenta que Manjarrés opinaba además que la idea debía envolverse de naturaleza, de observación; si no se hacía así, se podía caer en la abstracción que, como hombre del XIX, consideraba peligrosa (D-1896. Discursos (per anys) 1859-1915, RACBASJ). Manjarrés, figura apenas estudiada en la actualidad, fue uno de los profesores más influyentes y longevos de la Escuela, con un planteamiento estético que derivó del romanticismo nazareno al positivismo. El funcionamiento de sus clases, en las que se formaron dos generaciones de escultores, es decididamente parecido al actual, llegando hasta a preparar un libro de texto para su materia, buscando así evitar que los alumnos se concentrasen sólo en tomar apuntes y pudieran prestar más atención a las clases (Tarragó, 2012).

\section{A modo de conclusión}

El peso del dibujo y de lo clásico en la educación artística de la Escuela de Bellas Artes durante el siglo XIX resulta innegable, y el ámbito de la escultura no es una excepción. A pesar de las diferencias entre los planteamientos de sus distintos profesores - por otra parte, lógicas y necesarias -, éstos estan de acuerdo en la idiosincrasia de la aproximación a la práctica artística que preconizan: la necesidad de conjugar naturaleza y tradición, experiencia y modelo, observación y clásico. La Academia de Bellas Artes, a cargo de la Escuela a lo largo de buena parte del mil ochocientos, contribuye a hacer de dichos elementos una constante, apreciable no sólo en el campo de la docencia, sino también en la práctica artística en general, hecho que da razón de la extensión y duración del academicismo estético. A medida que se acerca el siglo $\mathrm{XX}$, la penetración de nuevas corrientes artísticas procedentes de Europa se hace patente en la Escuela, dando lugar a nuevas praxis que se transmiten, a su vez, a los escultores allí formados; un proceso que sentó las bases para que, ya en el siglo XX, fuera más común y aceptada la disensión de criterios y la libertad pedagógica entre los docentes en Bellas Artes.

En lo tocante a la dinámica de las clases, pocas fueron las modificaciones a las que se ve sometida la asignatura de Escultura durante la segunda mitad del XIX. El sistema de división de ésta en distintos niveles, siendo la copia del antiguo el primero y básico, no varía substancialmente a lo largo de este período. Sí lo hace la disciplina escolástica, que consigue adaptarse a los nuevos tiempos y a las nuevas relaciones entre el alumnado y el profesorado, pese a mantener siempre una rigidez que hoy es poco habitual en la educación superior.

A través de la fundación de las Academias Provinciales de Bellas Artes, en 1849, se pretende modernizar y uniformizar la enseñanza oficial de las disciplinas artísticas. 
En el caso de la escultura, los cambios significativos se producen ya a finales de siglo, con la nueva importancia otorgada al trabajo del natural y la apertura de los talleres, aunque los alumnos de la Escuela pudieron beneficiarse de la renovación de las enseñanzas en el marco de las asignaturas teóricas que cursaban en paralelo a Escultura. 1900 supone un paso más hacia adelante en lo tocante a la modernización del sistema educativo de las artes en Barcelona, un momento en el que la escuela se desvincula definitivamente de la Academia, aparecen asignaturas nuevas y se revisa la estructura curricular de las asignaturas, la relación de éstas entre ellas, el rol de los profesores, la normativa general de la institución, etc., adaptándose al siglo XX y posibilitando los cambios mayores que se producen en este campo a partir de la década de 1970, con la incorporación de los estudios al sistema educativo universitario.

\section{Referencias}

Academia Provincial de Bellas Artes de Barcelona (1897), Inventario general de 30 de junio de 1897, Archivo de la Reial Acadèmia Catalana de Belles Arts de Sant Jordi, Barceloona (RACBASJ).

Caja Museu, dipòsits, donacions, inventaris, expedients 1992-93 (RACBASJ)

Cid Priego, C. (1998). La vida y la obra del escultor neoclàsico catalán Damián Campeny i Estrany. Barcelona: Biblioteca de Catalunya.

D-1863. Discursos (per anys) 1859-1915, RACBASJ.

D-1896. Discursos (per anys) 1859-1915, RACBASJ.

Escuela Superior de Artes é Industrias y Bells Artes de Barcelona (1903), Memoria de su reorganizacion que cumpliendo el reglamento eleva el Ilustrisimo Señor subsecretario de Instruccion publica y Bellas Artes el director de la Escuela D. Leopoldo Soler y Perez. Barcelona: Imprenta de Henrich y Cia.

Faraudo, G. (1852). Estudios de la Historia natural del hombre aplicados á la pintura y escultura ó sea Lecciones de Anatomá y fisiología artísticas dadas en la Academia de Bellas Artes de Barcelona, Barcelona: Pons y Cía.

Freixa i Serra, M. (2008). En el decurs del Discurs. Una aproximació a la història del pensament estètic a l'Acadèmia de Belles Arts, 1856-1904. Barcelona: Arts Gràfiques Alpres.

Martinell, C. (1951). La Escuela de la Lonja en la vida artística barcelonesa. Barcelona: Escuela de Artes y Oficios artísticos de Barcelona

Mns. 251.1.6, RACBASJ.

Mns. 295.2.1.19, RACBASJ.

Mns. 295.2.2.2, RACBASJ.

Mns. 296.3.24, RACBASJ.

Mns. 75.10.5, RACBASJ.

Plano 3250D, RACBASJ.

Reglamento de la Escuela Gratuita establecida en la ciudad de Barcelona á espensas de la Junta de Comercio. Dispuesto y Mandado observar por la misma (1839). Barcelona: Herederos de Roca.

Rodríguez Codolà, M (1946), Venancio y Agapito Vallmitjana Barbany, Barcelona: Dirección General de Bellas Artes. 
Rodríguez Samaniego, C.; Esquinas, N. (2012), El Monumento al General dominicano Ulises Heureaux, de Pere Carbonell (1897). Comunicación. CEHA 2012. XIX Congreso Nacional de Historia del Arte CEHA: las Artes y la Arquitectura del Poder. Sección III: Los rostros de poder.

Ruiz Ortega, M. (1999). La escuela gratuita de diseño de Barcelona. 1775-1808. Barcelona: Biblioteca de Catalunya. Unitat Gràfica.

Subirachs i Burgaya, J. (1994), L'escultura catalana del segle XIX a Catalunya. Del Romanticisme al Realisme. Barcelona: Publicacions de l'Abadia de Montserrat: 27-52.

Tarragó, G. (2012). Romanticisme i positivisme en l'obra de José de Manjarrés, trabajo de final de Máster, Departamento de Historia del Arte, Universitat de Barcelona. 